\title{
Boundedness of higher order Riesz transforms associated with Schrödinger type operator on generalized Morrey spaces
}

\author{
Bijun Ren ${ }^{\mathrm{a}}$, Hui Wang ${ }^{\mathrm{b}, *}$ \\ ${ }^{a}$ Department of Information Engineering, Henan Institute of Finance and Banking, Zhengzhou, 451464, P. R. China. \\ ${ }^{b}$ Teachers College, Nanyang Institute of Technology, Nanyang, 473000, P. R. China.
}

Communicated by $\mathrm{Y}$. Hu

\begin{abstract}
Let $\mathcal{L}_{2}=(-\Delta)^{2}+V^{2}$ be a Schrödinger type operator, where $V \neq 0$ is a non-negative potential and belongs to the reverse Hölder class $\mathrm{RH}_{\mathrm{q}}$ for $\mathrm{q} \geqslant \mathrm{n} / 2, \mathrm{n} \geqslant 5$. The higher Riesz transform associated with $\mathcal{L}_{2}$ is denoted by $\mathcal{R}=\nabla^{2} \mathcal{L}_{2}^{-\frac{1}{2}}$ and its dual is denoted by $\mathcal{R}^{*}=\mathcal{L}_{2}^{-\frac{1}{2}} \nabla^{2}$. In this paper, we investigate the boundedness of higher Riesz transforms and their commutators on the generalized Morrey spaces related to some non-negative potential. (C)2017 All rights reserved.
\end{abstract}

Keywords: Schrödinger operator, Riesz transform, commutator, BMO, generalized Morrey space. 2010 MSC: 42B35, 35J10.

\section{Introduction}

Let us consider the Schrödinger type operator

$$
\mathcal{L}_{2}=(-\Delta)^{2}+V^{2}, \quad \text { on } \mathbb{R}^{n}, \quad n \geqslant 5,
$$

where $V$ is non-negative, $V \neq 0$, and belongs to the reverse Hölder class $R H_{q}$ for some $q \geqslant n / 2$, i.e., there exists a constant $C$ such that

$$
\left(\frac{1}{|B|} \int_{B} V(y)^{q} d y\right)^{1 / q} \leqslant \frac{C}{|B|} \int_{B} V(y) d y
$$

for every ball $\mathrm{B} \subset \mathbb{R}^{\mathrm{n}}$.

Obviously, $\mathrm{RH}_{\mathrm{q}_{2}} \subset \mathrm{RH}_{\mathrm{q}_{1}}$, if $\mathrm{q}_{2}>\mathrm{q}_{1}$. The most important property of the class $\mathrm{RH}_{\mathrm{q}}$ is its selfimprovement, that is, if $\mathrm{V} \in \mathrm{RH}_{\mathrm{q}}$, then $\mathrm{V} \in \mathrm{RH}_{\mathrm{q}+\epsilon}$ for some $\epsilon>0$. We define the reverse Hölder index of $\mathrm{V}$ as $\mathrm{q}_{0}=\sup \left\{\mathrm{q}: \mathrm{V} \in \mathrm{RH} \mathrm{H}_{\mathrm{q}}\right\}$.

The higher Riesz transform associated with $\mathcal{L}_{2}$ is defined by $\mathcal{R}=\nabla^{2} \mathcal{L}_{2}^{-1 / 2}$, and its dual is defined by $\mathcal{R}^{*}=\mathcal{L}_{2}^{-1 / 2} \nabla^{2}$. The $\mathrm{L}^{\mathrm{p}}$ boundedness of the higher Riesz transforms has been obtained in [4] by Liu and Dong.

\footnotetext{
*Corresponding author

Email addresses: renbijun1959@163.com (Bijun Ren), wanghui1639@126.com (Hui Wang)
} 
Theorem 1.1. Suppose $\mathrm{V} \in \mathrm{RH}_{\mathrm{q}}$ with $\frac{\mathrm{n}}{2} \leqslant \mathrm{q}<\mathrm{n}$. Let $\frac{1}{\mathrm{p}_{0}}=\frac{2}{\mathrm{q}_{0}}-\frac{2}{\mathrm{n}}, \mathrm{p}_{0}^{\prime}=\frac{\mathrm{p}_{0}}{\mathrm{p}_{0}-1}$.

(i) If $1<\mathrm{p}<\mathrm{p}_{0}$, then for all $\mathrm{f} \in \mathrm{L}^{\mathrm{p}}\left(\mathbb{R}^{\mathfrak{n}}\right)$

$$
\|\mathcal{R} f\|_{L^{p}\left(\mathbb{R}^{n}\right)} \leqslant C\|f\|_{L^{p}\left(\mathbb{R}^{n}\right)} .
$$

(ii) If $\mathrm{p}_{0}^{\prime}<\mathrm{p}<\infty$, then for all $\mathrm{f} \in \mathrm{L}^{\mathrm{p}}\left(\mathbb{R}^{\mathrm{n}}\right)$

$$
\left\|\mathcal{R}^{*} \mathbf{f}\right\|_{L^{p}\left(\mathbb{R}^{n}\right)} \leqslant C\|f\|_{L^{p}\left(\mathbb{R}^{n}\right)} .
$$

As in [10], for a given potential $V \in R H_{q}$ with $q \geqslant n / 2$, we define the auxiliary function

$$
\rho(x)=\sup \left\{r>0: \frac{1}{r^{n-2}} \int_{B(x, r)} V(y) d y \leqslant 1\right\}, x \in \mathbb{R}^{n} .
$$

It is well-known that $0<\rho(x)<\infty$ for any $x \in \mathbb{R}^{n}$.

According to [1], the new $\mathrm{BMO}$ space $\mathrm{BMO}_{\theta}(\rho)$ with $\theta \geqslant 0$ is defined as a set of all locally integrable functions $b$ such that

$$
\frac{1}{|B(x, r)|} \int_{B(x, r)}\left|b(y)-b_{B}\right| d y \leqslant C\left(1+\frac{r}{\rho(x)}\right)^{\theta},
$$

for all $x \in \mathbb{R}^{n}$ and $r>0$, where $b_{B}=\frac{1}{|B|} \int_{B} b(y) d y$. A norm for $b \in B M O_{\theta}(\rho)$, denoted by [b] $]_{\theta}$, is given by the infimum of the constants in the inequalities above.

Consider the commutators associated with the higher Riesz transforms and $b \in \mathrm{BMO}_{\theta}(\rho)$, Liu et al. in [6] showed that the commutators $[b, \mathcal{R}]$ and $\left[b, \mathcal{R}^{*}\right]$ are also bounded on $L^{p}\left(\mathbb{R}^{n}\right)$.

Theorem 1.2. Suppose $\mathrm{V} \in \mathrm{RH}_{\mathrm{q}}$ with $\frac{\mathrm{n}}{2} \leqslant \mathrm{q}<\mathrm{n}$. Let $\mathrm{b} \in \mathrm{BMO}_{\theta}(\rho), \frac{1}{\mathrm{p}_{0}}=\frac{2}{\mathrm{q}_{0}}-\frac{2}{\mathrm{n}}$ and $\mathrm{p}_{0}^{\prime}=\frac{\mathrm{p}_{0}}{\mathrm{p}_{0}-1}$.

(i) If $1<\mathrm{p}<\mathrm{p}_{0}$, then for all $\mathrm{f} \in \mathrm{L}^{\mathrm{p}}\left(\mathbb{R}^{\mathrm{n}}\right)$

$$
\|[b, \mathcal{R}] f\|_{L^{p}\left(\mathbb{R}^{n}\right)} \leqslant C[b]_{\theta}\|f\|_{L^{p}\left(\mathbb{R}^{n}\right)} .
$$

(ii) If $\mathrm{p}_{0}^{\prime}<\mathrm{p}<\infty$, then for all $\mathrm{f} \in \mathrm{L}^{\mathrm{p}}\left(\mathbb{R}^{\mathrm{n}}\right)$

$$
\left\|\left[b, \mathcal{R}^{*}\right] f\right\|_{L^{p}\left(\mathbb{R}^{n}\right)} \leqslant C[b]_{\theta}\|f\|_{L^{p}\left(\mathbb{R}^{n}\right)} .
$$

The classical Morrey space was introduced by Morrey in [8], since then a large number of investigations have been given to them by mathematicians. Recently, many authors established the boundedness of some Schrödinger type operators on the Morrey spaces related to the nonnegative potential $\mathrm{V}$ belonging to the reverse Hölder class (see $[2,5,9,11]$ ). Motivated by these results, our aim in this paper is to establish the boundedness for the higher Riesz transforms associated with Schrödinger operators and their commutators on generalized Morrey spaces related to the certain non-negative potentials.

We now introduce the definition of generalized Morrey spaces related to the nonnegative potential $\mathrm{V}$.

Definition 1.3. Let $\varphi(x, r)$ be a positive measurable function on $\mathbb{R}^{n} \times(0, \infty), 1 \leqslant p<\infty, \alpha \geqslant 0$, and $\mathrm{V} \in \mathrm{RH}_{\mathrm{q}}$. We denote by $M_{p, \varphi}^{\alpha, V}=M_{p, \varphi}^{\alpha, V}\left(\mathbb{R}^{n}\right)$ the generalized Morrey space related to the non-negative potential $V$, that is, the space of all functions $f \in L_{\text {loc }}^{p}\left(\mathbb{R}^{n}\right)$ with finite quasinorm

$$
\|f\|_{M_{\mathcal{p}, \varphi}^{\alpha, V}}=\sup _{x \in \mathbb{R}^{n}, r>0}\left(1+\frac{r}{\rho(x)}\right)^{\alpha} \varphi(x, r)^{-1} r^{-n / p}\|f\|_{L^{p}(B(x, r))} .
$$

\section{Remark 1.4.}

(i) When $\alpha=0$, and $\varphi(x, r)=r^{\lambda-n / p}, M_{p, \varphi}^{\alpha, V}\left(\mathbb{R}^{n}\right)$ is the classical Morrey space $L^{p, \lambda}\left(\mathbb{R}^{n}\right)$ introduced in [8]; 
(ii) when $\alpha=0$, and $\varphi(x, r)^{p}=\Phi(r) r^{-n}, M_{p, \varphi}^{\alpha, V}\left(\mathbb{R}^{n}\right)$ is the generalized Morrey space $L^{p, \Phi}\left(\mathbb{R}^{n}\right)$ (see [7]);

(iii) when $\varphi(x, r)=r^{-n / q}, M_{p, \varphi}^{\alpha, V}\left(\mathbb{R}^{n}\right)$ is the Morrey space associated with Schrödinger operator $M_{p, V}^{q, \alpha}\left(\mathbb{R}^{n}\right)$ studied in $[2,11]$.

Our main results in this paper are formulated as follows.

Theorem 1.5. Let $\mathrm{V} \in \mathrm{RH}_{\mathrm{q}}$ with $\frac{\mathrm{n}}{2} \leqslant \mathrm{q}<\mathrm{n}$, let $\alpha \geqslant 0$, and let $\frac{1}{\mathrm{p}_{0}}=\frac{2}{\mathrm{q}_{0}}-\frac{2}{\mathrm{n}}, \mathrm{q}_{0}$ is the reverse Hölder index of $\mathrm{V}$. Suppose $\left(\varphi_{1}, \varphi_{2}\right)$ satisfies the condition

$$
\int_{r}^{\infty} \frac{\underset{t<s<\infty}{\operatorname{essinf}} \varphi_{1}(x, s) s^{\frac{n}{p}}}{t^{\frac{n}{p}}} \frac{d t}{t} \leqslant c_{0} \varphi_{2}(x, r),
$$

where $\mathrm{c}_{0}$ does not depend on $\mathrm{x}$ and $\mathrm{r}$.

(i) If $1<p<p_{0}$, then

$$
\|\mathcal{R} f\|_{M_{p, \varphi_{2}}^{\alpha, V}} \leqslant C\|f\|_{M_{p, \varphi_{1}}^{\alpha, V}}
$$

(ii) If $\mathrm{p}_{0}^{\prime}<\mathrm{p}<\infty$, then

$$
\left\|\mathcal{R}^{*} f\right\|_{M_{p, \varphi_{2}}^{\alpha, V}} \leqslant C\|f\|_{M_{p, \varphi_{1}}^{\alpha, V}}
$$

Theorem 1.6. Let $\mathrm{V} \in \mathrm{RH}_{\mathrm{q}}$ with $\frac{\mathrm{n}}{2} \leqslant \mathrm{q}<\mathrm{n}$, let $\alpha \geqslant 0$, and let $\frac{1}{\mathrm{p}_{0}}=\frac{2}{\mathrm{q}_{0}}-\frac{2}{\mathrm{n}}$. Suppose $\mathrm{b} \in \mathrm{BMO}_{\theta}(\rho)$ and $\left(\varphi_{1}, \varphi_{2}\right)$ satisfies the condition

$$
\int_{r}^{\infty}\left(1+\ln \frac{t}{r}\right) \frac{\substack{\operatorname{essinf} \varphi_{t}<s \\ t<\infty}}{t^{\frac{n}{p}}} \frac{s^{\frac{n}{p}}}{t} \leqslant c_{0} \varphi_{2}(x, r),
$$

where $\mathrm{c}_{0}$ does not depend on $\mathrm{x}$ and $\mathrm{r}$.

(i) If $1<p<p_{0}$, then

$$
\|[b, \mathcal{R}] f\|_{M_{p, \varphi_{2}}^{\alpha, V}} \leqslant C[b]_{\theta}\|f\|_{M_{p, \varphi_{1}}^{\alpha, V}}
$$

(ii) If $\mathrm{p}_{0}^{\prime}<\mathrm{p}<\infty$, then

$$
\left\|\left[b, \mathcal{R}^{*}\right] f\right\|_{M_{p, \varphi_{2}}^{\alpha, V}} \leqslant \mathrm{C}[\mathrm{b}]_{\theta}\|f\|_{M_{p, \varphi_{1}}^{\alpha, V}} .
$$

In this paper, we shall use the symbol $A \lesssim B$ to indicate that there exists a universal positive constant $C$, independent of all important parameters, such that $A \leqslant C B . A \approx B$ means that $A \lesssim B$ and $B \lesssim A$.

\section{Some preliminaries}

We would like to recall the important properties concerning the auxiliary function.

Lemma 2.1 ([3]). Let $\mathrm{V} \in \mathrm{RH}_{\mathrm{n} / 2}$. Then there exists constants $\mathrm{C}>0$ and $\mathrm{l}_{0}>0$ such that

$$
\frac{1}{r^{n-2}} \int_{B(x, r)} V(y) d y \leqslant C\left(1+\frac{r}{\rho(x)}\right)^{l_{0}} .
$$


Lemma 2.2 ([1]). Let $\mathrm{V} \in \mathrm{RH}_{\mathrm{n} / 2}$. For the auxiliary function $\rho$ there exist $\mathrm{C}$ and $\mathrm{k}_{0} \geqslant 1$ such that

$$
C^{-1} \rho(x)\left(1+\frac{|x-y|}{\rho(x)}\right)^{-k_{0}} \leqslant \rho(y) \leqslant C \rho(x)\left(1+\frac{|x-y|}{\rho(x)}\right)^{\frac{k_{0}}{1+k_{0}}},
$$

for all $x, y \in \mathbb{R}^{n}$.

Lemma 2.3. Suppose $\mathrm{x} \in \mathrm{B}\left(\mathrm{x}_{0}, \mathrm{r}\right)$. Then for $\mathrm{k} \in \mathbb{N}$ we have

$$
\frac{1}{\left(1+\frac{2^{k} r}{\rho(x)}\right)^{N}} \lesssim \frac{1}{\left(1+\frac{2^{k} r}{\rho\left(x_{0}\right)}\right)^{N /\left(k_{0}+1\right)}}
$$

Proof. By Lemma 2.2 we have

$$
\begin{aligned}
\frac{1}{\left(1+\frac{2^{k_{r}}}{\rho(x)}\right)^{N}} & \lesssim \frac{1}{\left(1+\frac{2^{k_{r}}}{\rho\left(x_{0}\right)\left(1+\frac{\left|x-x_{0}\right|}{\rho\left(x_{0}\right)}\right)^{\frac{k_{0}}{k_{0}+1}}}\right)^{N}} \\
& \lesssim \frac{\left(1+\frac{\left|x-x_{0}\right|}{\rho\left(x_{0}\right)}\right)^{\frac{k_{0} N}{k_{0}+1}}}{\left(1+\frac{2^{k} r}{\rho\left(x_{0}\right)}\right)^{N}} \\
& \lesssim \frac{1}{\left(1+\frac{2^{k} r}{\rho\left(x_{0}\right)}\right)^{N /\left(k_{0}+1\right)}} .
\end{aligned}
$$

We give an inequality about the new $\mathrm{BMO}$ space $\mathrm{BMO}_{\theta}(\rho)$.

Lemma 2.4 ([4]). Let $1 \leqslant s<\infty, \mathrm{b} \in \mathrm{BMO}_{\theta}(\rho)$, and $\mathrm{B}=\mathrm{B}(x, r)$. Then

$$
\left(\frac{1}{\left|2^{k} B\right|} \int_{2^{k} B}\left|b(y)-b_{B}\right|^{s} d y\right)^{1 / s} \leqslant[b]_{\theta} k\left(1+\frac{2^{k} r}{\rho(x)}\right)^{\theta^{\prime}},
$$

for all $\mathrm{k} \in \mathbb{N}$, with $\theta^{\prime}=\left(\mathrm{k}_{0}+1\right) \theta$ and $\mathrm{k}_{0}$ is the constant appearing in Lemma 2.2.

Let $\mathrm{K}^{*}$ be the kernel of $\mathcal{R}^{*}$, we have

Lemma 2.5 ([6]). Suppose $\mathrm{V} \in \mathrm{RH}_{\mathrm{q}}$, we have the following results.

(i) If $\frac{\mathrm{n}}{2} \leqslant \mathrm{q}<\mathrm{n}$, then for every $\mathrm{N}$, there exists a constant $\mathrm{C}_{\mathrm{N}}>0$ such that

$$
\left|\mathrm{K}^{*}(x, z)\right| \leqslant \frac{\mathrm{C}_{\mathrm{N}}\left(1+\frac{|x-z|}{\rho(x)}\right)^{-\mathrm{N}}}{|x-z|^{\mathrm{n}-2}}\left(\int_{\mathrm{B}(z,|x-z| / 4)} \frac{\mathrm{V}^{2}(\mathrm{u})}{|u-z|^{\mathrm{n}-2}} \mathrm{du}+\frac{1}{|x-z|^{2}}\right) .
$$

(ii) When $\mathrm{q} \geqslant \mathrm{n}$, the term involving $\mathrm{V}$ can be dropped from above formula.

Let $\mathrm{K}$ be the kernel of $\mathcal{R}$, then $\mathrm{K}$ has exactly the same estimates of $\mathrm{K}^{*}$, see [6].

Finally, we recall a relationship between essential supremum and essential infimum.

Lemma 2.6 ([12]). Let $\mathrm{f}$ be a real-valued non-negative function and measurable on $\mathrm{E}$. Then

$$
(\underset{x \in E}{\operatorname{essinf}} f(x))^{-1}=\underset{x \in E}{\operatorname{ess} \sup } \frac{1}{f(x)}
$$




\section{Proof of Theorem 1.5}

To prove Theorem 1.5, we first investigate the following local estimates.

Theorem 3.1. Let $\mathrm{V} \in \mathrm{RH}_{\mathrm{q}}$ with $\frac{\mathrm{n}}{2} \leqslant \mathrm{q}<\mathrm{n}$.

(i) If $1<\mathrm{p}<\mathrm{p}_{0}$, then for any $\mathrm{f} \in \mathrm{L}_{\text {loc }}^{\mathrm{p}}\left(\mathbb{R}^{\mathrm{n}}\right)$ we have

$$
\|\mathcal{R}(f)\|_{L^{p}\left(B\left(x_{0}, r\right)\right)} \lesssim r^{\frac{n}{p}} \int_{2 r}^{\infty} \frac{\|f\|_{L^{p}\left(B\left(x_{0}, t\right)\right)}}{t^{\frac{n}{p}}} \frac{d t}{t} ;
$$

(ii) if $\mathrm{p}_{0}^{\prime}<\mathrm{p}<\infty$, then for any $\mathrm{f} \in \mathrm{L}_{\text {loc }}^{\mathrm{p}}\left(\mathbb{R}^{\mathrm{n}}\right)$ we have

$$
\left\|\mathcal{R}^{*}(f)\right\|_{L^{p}\left(B\left(x_{0}, r\right)\right)} \lesssim r^{\frac{n}{p}} \int_{2 r}^{\infty} \frac{\|f\|_{L^{p}\left(B\left(x_{0}, t\right)\right)}}{t^{\frac{n}{p}}} \frac{d t}{t} .
$$

Proof. Since the proofs for the case $1<p<p_{0}$ and the case $p_{0}^{\prime}<p<\infty$ are very similar, we only prove the case $p_{0}^{\prime}<p<\infty$.

For arbitrary $\chi_{0} \in \mathbb{R}^{n}$, set $B=B\left(\chi_{0}, r\right)$. We write $f$ as $f=f_{1}+f_{2}$, where $f_{1}(y)=f(y) \chi_{B}\left(x_{0}, 2 r\right)(y)$. Then

$$
\left\|\mathcal{R}^{*}(f)\right\|_{L^{p}\left(B\left(x_{0}, r\right)\right)} \leqslant\left\|\mathcal{R}^{*}\left(f_{1}\right)\right\|_{L^{p}\left(B\left(x_{0}, r\right)\right)}+\left\|\mathcal{R}^{*}\left(f_{2}\right)\right\|_{L^{p}\left(B\left(x_{0}, r\right)\right)} .
$$

Since $f_{1} \in L^{p}\left(\mathbb{R}^{n}\right)$, then by the boundedness of $\mathcal{R}^{*}$ on $L^{p}\left(\mathbb{R}^{\mathfrak{n}}\right)\left(p_{0}^{\prime}<p<\infty\right)$, it follows that

$$
\begin{aligned}
\left\|\mathcal{R}^{*}\left(f_{1}\right)\right\|_{L^{p}\left(B\left(x_{0}, r\right)\right)} & \lesssim\|f\|_{L^{p}\left(B\left(x_{0}, 2 r\right)\right)} \\
& \lesssim r^{\frac{n}{p}}\|f\|_{L^{p}\left(B\left(x_{0}, 2 r\right)\right)} \int_{2 r}^{\infty} \frac{d t}{t^{\frac{n}{p}+1}} \\
& \lesssim r^{\frac{n}{p}} \int_{2 r}^{\infty} \frac{\|f\|_{L^{p}\left(B\left(x_{0}, t\right)\right)}}{t^{\frac{n}{p}}} \frac{d t}{t} .
\end{aligned}
$$

To estimate $\left\|\mathcal{R}^{*}\left(f_{2}\right)\right\|_{L^{p}\left(B\left(x_{0}, r\right)\right)}$, obverse that $x \in B, y \in(2 B)^{c}$ implies $|x-y| \approx\left|x_{0}-y\right|$. Then by Lemma 2.5 we have

$$
\begin{aligned}
\sup _{x \in B\left(x_{0}, r\right)}\left|\mathcal{R}^{*}\left(f_{2}\right)(x)\right| \leqslant & \int_{(2 B) c}\left|K^{*}(x, y) f(y)\right| d y \\
& \lesssim \int_{(2 B) c} \frac{1}{\left(1+\frac{\left|x_{0}-y\right|}{\rho(x)}\right)^{N}} \frac{|f(y)|}{\left|x_{0}-y\right|^{n}} d y \\
& +\int_{(2 B) c} \frac{1}{\left(1+\frac{\left|x_{0}-y\right|}{\rho(x)}\right)^{N}} \frac{|f(y)|}{\left|x_{0}-y\right|^{n-2}} \int_{B\left(y,\left|x_{0}-y\right| / 4\right)} \frac{V^{2}(z)}{|z-y|^{n-2}} d z d y \\
= & K_{1}+K_{2} .
\end{aligned}
$$

By splitting into annuli and Hölder's inequality we get

$$
\begin{aligned}
K_{1} & \lesssim \frac{1}{\left(1+\frac{2 r}{\rho(x)}\right)^{N}} \sum_{k=1}^{\infty}\left(2^{k+1} r\right)^{-n} \int_{2^{k+1} B}|f(y)| d y \\
& \lesssim \frac{1}{\left(1+\frac{2 r}{\rho(x)}\right)^{N}} \sum_{k=1}^{\infty}\left(2^{k+1} r\right)^{-\frac{n}{p}}\|f\|_{L^{p}\left(B\left(x_{0}, 2^{k+1} r\right)\right)}
\end{aligned}
$$

Then, by Lemma 2.3 we have

$$
\mathrm{K}_{1} \lesssim \frac{1}{\left(1+\frac{2 \mathrm{r}}{\rho(x)}\right)^{N}} \sum_{k=1}^{\infty}\left(2^{k+1} \mathrm{r}\right)^{-1-\frac{\mathfrak{n}}{\mathrm{p}}}\|f\|_{L^{p}\left(B\left(x_{0}, 2^{k+1} r\right)\right)} \int_{2^{k} r}^{2^{k+1} r} d t
$$




$$
\lesssim \frac{1}{\left(1+\frac{2 r}{\rho\left(x_{0}\right)}\right)^{N /\left(k_{0}+1\right)}} \int_{2 r}^{\infty} \frac{\|f\|_{L^{p}\left(B\left(x_{0}, t\right)\right)}}{t^{\frac{n}{p}}} \frac{d t}{t}
$$

For $\mathrm{K}_{2}$, by splitting into annuli, Lemma 2.3 and Hölder's inequality we get

$$
\begin{aligned}
& \mathrm{K}_{2} \lesssim \sum_{\mathrm{k}=1}^{\infty} \frac{1}{\left(2^{\mathrm{k}+1} \mathrm{r}\right)^{\mathrm{n}-2}} \frac{1}{\left(1+\frac{2^{\mathrm{k}} \mathrm{r}}{\rho(\mathrm{x})}\right)^{\mathrm{N}}} \int_{2^{\mathrm{k}+1} \mathrm{~B}}|\mathrm{f}(\mathrm{y})| \int_{\mathrm{B}\left(\mathrm{x}_{0}, 2^{\mathrm{k}+3} \mathrm{r}\right)} \frac{\mathrm{V}^{2}(z)}{|z-y|^{n-2}} \mathrm{~d} z \mathrm{~d} \mathrm{y} \\
& \lesssim \sum_{k=1}^{\infty} \frac{1}{\left(2^{k+1} r\right)^{n-2}} \frac{1}{\left(1+\frac{2^{k} r}{\rho\left(x_{0}\right)}\right)^{N /\left(k_{0}+1\right)}} \int_{2^{k+1} B}|f(y)| I_{2}\left(V^{2} \chi_{B}\left(x_{0}, 2^{k+3} r\right)(y) d y\right. \\
& \lesssim \sum_{k=1}^{\infty} \frac{1}{\left(2^{k+1} r\right)^{n-2}} \frac{1}{\left(1+\frac{2^{k} r}{\rho\left(x_{0}\right)}\right)^{N /\left(k_{0}+1\right)}}\|f\|_{L^{p}\left(B\left(x_{0}, 2^{k+1} r\right)\right)}\left\|I_{2}\left(V^{2} \chi_{B\left(x_{0}, 2^{k+3} r\right)}\right)\right\|_{L^{p^{\prime}\left(\mathbb{R}^{n}\right)},}
\end{aligned}
$$

where $I_{2}(f)(y)=\int_{R^{n}} \frac{f(z)}{|z-y|^{n-2}} d z$ is the Riesz potential. Since $p_{0}^{\prime}<p<\infty, 1 / p_{0}=2 / q_{0}-2 / n$, we can select an appropriate number s such that $1 / p^{\prime}=2 / s-2 / n$. Note that $I_{2}$ is bounded from $L^{s / 2}$ to $L^{p^{\prime}}$, and $\mathrm{V} \in \mathrm{RH}_{\mathrm{s}}$, then by Lemma 2.1 we get

$$
\begin{aligned}
& \left\|I_{2}\left(V^{2} \chi_{B\left(\chi_{0}, 2^{k+3} r\right)}\right)\right\|_{L^{p^{\prime}}\left(\mathbb{R}^{n}\right)} \lesssim\left\|V^{2} \chi_{B\left(\chi_{0}, 2^{k+3} r\right)}\right\|_{L^{s / 2}\left(\mathbb{R}^{n}\right)} \\
& =\left|B\left(x_{0}, 2^{k+3} r\right)\right|^{\frac{2}{s}}\left(\frac{1}{\left|B\left(x_{0}, 2^{k+3} r\right)\right|} \int_{B\left(x_{0}, 2^{k+1} r\right)} V^{s}(z) d z\right)^{2 / s} \\
& \lesssim\left|\mathrm{B}\left(x_{0}, 2^{\mathrm{k}+1} r\right)\right|^{\frac{2}{s}-\frac{4}{n}}\left(\frac{1}{\left(2^{k+3} r\right)^{n-2}} \int_{B\left(x_{0}, 2^{k+3} r\right)} V(z) d z\right)^{2} \\
& \lesssim\left(2^{k+1} r\right)^{\frac{n}{p^{\prime}}-2}\left(1+\frac{2^{k} r}{\rho\left(x_{0}\right)}\right)^{2 l_{0}} \text {. }
\end{aligned}
$$

Thus, we get

$$
\begin{aligned}
& \mathrm{K}_{2} \lesssim \sum_{\mathrm{k}=1}^{\infty}\left(2^{\mathrm{k}+1} \mathrm{r}\right)^{-\frac{\mathrm{n}}{\mathrm{p}}} \frac{1}{\left(1+\frac{2^{\mathrm{k}} \mathrm{r}}{\rho\left(\mathrm{x}_{0}\right)}\right)^{\left(\mathrm{N} /\left(\mathrm{k}_{0}+1\right)-2 \mathrm{l}_{0}\right)}}\|\mathrm{f}\|_{\mathrm{L}^{\mathrm{p}}\left(\mathrm{B}\left(\mathrm{x}_{0}, 2^{\mathrm{k}+1} \mathrm{r}\right)\right)} \\
& \lesssim \frac{1}{\left(1+\frac{2 \mathrm{r}}{\rho\left(\mathrm{x}_{0}\right)}\right)^{\left(\mathrm{N} /\left(\mathrm{k}_{0}+1\right)-2 \mathrm{l}_{0}\right)}} \sum_{\mathrm{k}=1}^{\infty}\left(2^{\mathrm{k}+1} \mathrm{r}\right)^{-\frac{\mathfrak{n}}{\mathrm{p}}}\|\mathrm{f}\|_{\mathrm{L}^{\mathrm{p}}\left(\mathrm{B}\left(\mathrm{x}_{0}, 2^{\mathrm{k}+1} \mathrm{r}\right)\right)}
\end{aligned}
$$

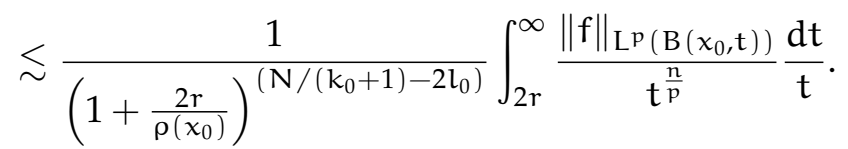

Combined with estimates of $K_{1}$ and $K_{2}$ we obtain

$$
\sup _{x \in B\left(x_{0}, r\right)}\left|\mathcal{R}^{*}\left(f_{2}\right)(x)\right| \lesssim \frac{1}{\left(1+\frac{2 r}{\rho\left(x_{0}\right)}\right)^{\left(N /\left(k_{0}+1\right)-2 l_{0}\right)}} \int_{2 r}^{\infty} \frac{\|f\|_{L^{p}\left(B\left(x_{0}, t\right)\right)}}{t^{\frac{n}{p}}} \frac{d t}{t} .
$$

Taking $N \geqslant 2 l_{0}\left(k_{0}+1\right)$, then

$$
\left\|\mathcal{R}^{*}\left(f_{2}\right)\right\|_{L^{p}\left(B\left(x_{0}, r\right)\right)} \lesssim r^{\frac{n}{p}} \int_{2 r}^{\infty} \frac{\|f\|_{L^{p}\left(B\left(x_{0}, t\right)\right)}}{t^{\frac{n}{p}}} \frac{d t}{t}
$$

This completes the proof of Theorem 3.1. 
Proof of Theorem 1.5. From Lemma 2.6, we have

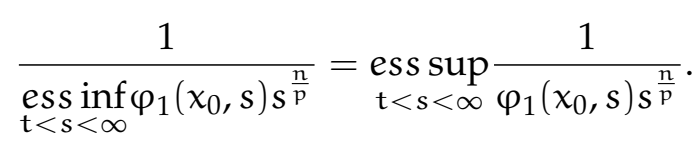

Note the fact that $\|f\|_{L^{p}\left(B\left(x_{0}, t\right)\right)}$ is a nondecreasing function of $t$, and $f \in M_{p^{\prime}, \varphi_{1}}^{\alpha, V}$, then

$$
\begin{aligned}
\frac{\left(1+\frac{t}{\rho\left(x_{0}\right)}\right)^{\alpha}\|f\|_{L^{p}\left(B\left(x_{0}, t\right)\right)}}{e_{t<s<\infty}^{e s s i n f} \varphi_{1}\left(x_{0}, s\right) s^{\frac{n}{p}}} & \lesssim \underset{t<s<\infty}{\operatorname{esssup}} \frac{\left(1+\frac{t}{\rho\left(x_{0}\right)}\right)^{\alpha}\|f\|_{L^{p}\left(B\left(x_{0}, t\right)\right)}}{\varphi_{1}\left(x_{0}, s\right) s^{\frac{n}{p}}} \\
& \lesssim \sup _{0<s<\infty} \frac{\left(1+\frac{s}{\rho\left(x_{0}\right)}\right)^{\alpha}\|f\|_{L^{p}\left(B\left(x_{0}, s\right)\right)}}{\varphi_{1}\left(x_{0}, s\right) s^{\frac{n}{p}}} \lesssim\|f\|_{M_{\mathcal{P}, \varphi_{1}}^{\alpha, V}}
\end{aligned}
$$

Since $\alpha \geqslant 0$, and $\left(\varphi_{1}, \varphi_{2}\right)$ satisfies the condition (1.1), we get

$$
\begin{aligned}
& \int_{2 r}^{\infty} \frac{\|f\|_{L^{p}\left(B\left(x_{0}, t\right)\right)}}{t^{\frac{n}{p}}} \frac{d t}{t}=\int_{2 r}^{\infty} \frac{\left(1+\frac{t}{\rho\left(x_{0}\right)}\right)^{\alpha}\|f\|_{L^{p}\left(B\left(x_{0}, t\right)\right)}}{\underbrace{e s s i n f}_{t<s<\infty} \varphi_{1}\left(x_{0}, s\right) s^{\frac{n}{p}}} \frac{\underset{t<s<\infty}{\operatorname{essinf} \varphi_{1}\left(x_{0}, s\right) s^{\frac{n}{p}}}}{\left(1+\frac{t}{\rho\left(x_{0}\right)}\right)^{\alpha} t^{\frac{n}{p}}} \frac{d t}{t}
\end{aligned}
$$

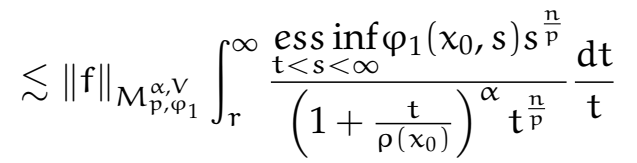

$$
\begin{aligned}
& \lesssim\|f\|_{M_{p, \varphi_{1}}^{\alpha, v}}\left(1+\frac{r}{\rho\left(x_{0}\right)}\right)^{-\alpha} \int_{r}^{\infty} \frac{\underset{t<s<\infty}{\operatorname{essinf}} \varphi_{1}\left(x_{0}, s\right) s^{\frac{n}{p}}}{t^{\frac{n}{p}}} \frac{d t}{t} \\
& \lesssim\|f\|_{M_{p, \varphi_{1}}^{\alpha, V}}\left(1+\frac{r}{\rho\left(x_{0}\right)}\right)^{-\alpha} \varphi_{2}\left(x_{0}, r\right) .
\end{aligned}
$$

Then by Theorem 3.1 we get

$$
\begin{aligned}
\left\|\mathcal{R}^{*}(f)\right\|_{M_{\mathcal{p}, \varphi_{2}}^{\alpha, v}} & \lesssim \sup _{x_{0} \in \mathbb{R}^{n}, r>0}\left(1+\frac{r}{\rho\left(x_{0}\right)}\right)^{\alpha} \varphi_{2}\left(x_{0}, r\right)^{-1} r^{-n / p}\left\|\mathcal{R}^{*}(f)\right\|_{L^{p}\left(B\left(x_{0}, r\right)\right)} \\
& \lesssim \sup _{x_{0} \in \mathbb{R}^{n}, r>0}\left(1+\frac{r}{\rho\left(x_{0}\right)}\right)^{\alpha} \varphi_{2}\left(x_{0}, r\right)^{-1} \int_{2 r}^{\infty} \frac{\|f\|_{L^{p}\left(B\left(x_{0}, t\right)\right)}}{t^{\frac{n}{p}}} \frac{d t}{t} \\
& \lesssim\|f\|_{M_{\mathcal{p}, \varphi_{1}}^{\alpha, V}}
\end{aligned}
$$

\section{Proof of Theorem 1.6}

Let us start with local estimates.

Theorem 4.1. Let $\mathrm{V} \in \mathrm{RH}_{\mathrm{q}}$ with $\frac{\mathrm{n}}{2} \leqslant \mathrm{q}<\mathrm{n}$, and let $\mathrm{b} \in \mathrm{BMO}_{\theta}(\rho)$. If $\mathrm{p}_{0}^{\prime}<\mathrm{p}<\infty$, then the inequality

$$
\left\|\left[b, \mathcal{R}^{*}\right](f)\right\|_{L^{p}\left(B\left(x_{0}, r\right)\right)} \lesssim[b]_{\theta} r^{\frac{n}{p}} \int_{2 r}^{\infty}\left(1+\ln \frac{t}{r}\right) \frac{\|f\|_{L^{p}\left(B\left(x_{0}, t\right)\right)}}{t^{\frac{n}{p}}} \frac{d t}{t},
$$

holds for any $\mathrm{f} \in \mathrm{L}_{\text {loc }}^{\mathrm{p}}\left(\mathbb{R}^{\mathrm{n}}\right)$. If $1<\mathrm{p}<\mathrm{p}_{0}$, then the inequality

$$
\|[b, \mathcal{R}](f)\|_{L^{p}\left(B\left(x_{0}, r\right)\right)} \lesssim[b]_{\theta} r^{\frac{n}{p}} \int_{2 r}^{\infty}\left(1+\ln \frac{t}{r}\right) \frac{\|f\|_{L^{p}\left(B\left(x_{0}, t\right)\right)}}{t^{\frac{n}{p}}} \frac{d t}{t}
$$

holds for any $\mathrm{f} \in \mathrm{L}_{\text {loc }}^{\mathrm{p}}\left(\mathbb{R}^{\mathrm{n}}\right)$. 
Proof. We write $f$ as $f=f_{1}+f_{2}$, where $f_{1}(y)=f(y) \chi_{B\left(x_{0}, 2 r\right)}(y)$. Then

$$
\left\|\left[b, \mathcal{R}^{*}\right](f)\right\|_{L^{p}\left(B\left(x_{0}, r\right)\right)} \leqslant\left\|\left[b, \mathcal{R}^{*}\right]\left(f_{1}\right)\right\|_{L^{p}\left(B\left(x_{0}, r\right)\right)}+\left\|\left[b, \mathcal{R}^{*}\right]\left(f_{2}\right)\right\|_{L^{p}\left(B\left(x_{0}, r\right)\right)} .
$$

By the boundedness of $\left[b, \mathcal{R}^{*}\right]$ on $L^{p}\left(\mathbb{R}^{\mathfrak{n}}\right)$ and similar to the estimate of (3.1) we get

$$
\begin{aligned}
\left\|\left[b, \mathcal{R}^{*}\right]\left(f_{1}\right)\right\|_{L^{p}\left(B\left(x_{0}, r\right)\right)} & \lesssim[b]_{\theta}\|f\|_{L^{p}\left(B\left(x_{0}, 2 r\right)\right)} \\
& \lesssim[b]_{\theta} r^{\frac{n}{p}} \int_{2 r}^{\infty}\left(1+\ln \frac{t}{r}\right) \frac{\|f\|_{L^{p}\left(B\left(x_{0}, t\right)\right)}}{t^{\frac{n}{p}}} \frac{d t}{t}
\end{aligned}
$$

We now turn to deal with the term $\left\|\left[b, \mathcal{R}^{*}\right]\left(f_{2}\right)\right\|_{L^{p}\left(B\left(x_{0}, r\right)\right)}$. For any given $x \in B\left(x_{0}, r\right)$ we have

$$
\left|\left[b, \mathcal{R}^{*}\right] f_{2}(x)\right| \leqslant\left|b(x)-b_{2 B}\right|\left|\mathcal{R}^{*}\left(f_{2}\right)(x)\right|+\left|\mathcal{R}^{*}\left(\left(b-b_{2 B}\right) f_{2}\right)(x)\right| .
$$

By (3.3) we have

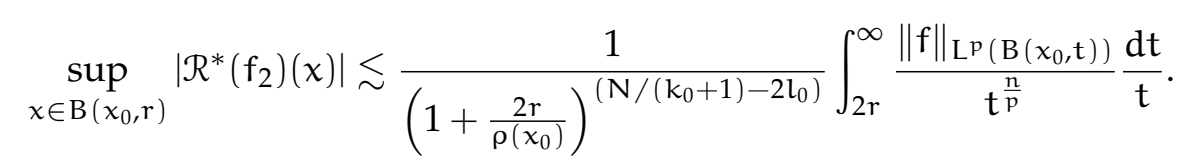

By Lemma 2.4,

$$
\left\|\mathrm{b}-\mathrm{b}_{2 \mathrm{~B}}\right\|_{\mathrm{L}^{\mathrm{p}}\left(\mathrm{B}\left(\mathrm{x}_{0}, \mathrm{r}\right)\right.} \lesssim[\mathrm{b}]_{\theta} \mathrm{r}^{\frac{\mathrm{n}}{\mathrm{p}}}\left(1+\frac{2 \mathrm{r}}{\rho\left(\mathrm{x}_{0}\right)}\right)^{\theta}
$$

Then, taking $N \geqslant\left(k_{0}+1\right)\left(\theta+2 l_{0}\right)$ we get

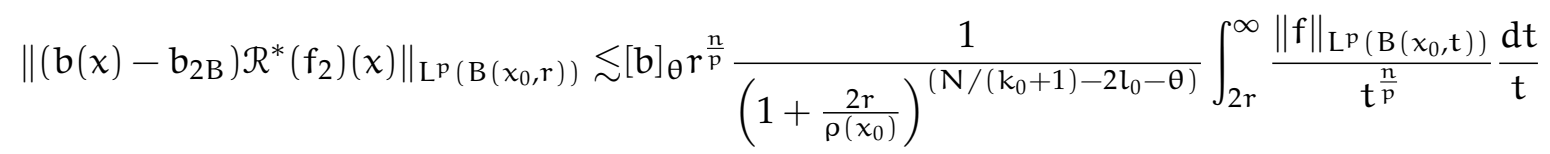

$$
\begin{aligned}
& \lesssim[b]_{\theta} r^{\frac{n}{p}} \int_{2 r}^{\infty}\left(1+\ln \frac{t}{r}\right) \frac{\|f\|_{L^{p}\left(B\left(x_{0}, t\right)\right)}}{t^{\frac{n}{p}}} \frac{d t}{t} .
\end{aligned}
$$

Let us estimate $\left\|\mathcal{R}^{*}\left(\left(b-b_{2 B}\right) f_{2}\right)\right\|_{L^{p}\left(B\left(x_{0}, r\right)\right)}$. By Lemma 2.5 we have

$$
\begin{aligned}
\sup _{x \in B\left(x_{0}, r\right)}\left|\mathcal{R}^{*}\left(\left(b-b_{2 B}\right) f_{2}\right)(x)\right| \leqslant & \int_{(2 B)^{c}}\left|K^{*}(x, y)\left(b(y)-b_{2 B}\right) f(y)\right| d y \\
\lesssim & \int_{(2 B)^{c}} \frac{\left|b(y)-b_{2 B}\right|}{\left(1+\frac{\left|x_{0}-y\right|}{\rho(x)}\right)^{N}} \frac{|f(y)|}{\left|x_{0}-y\right|^{n}} d y \\
& +\int_{(2 B)^{c}} \frac{\left|b(y)-b_{2 B}\right|}{\left(1+\frac{\left|x_{0}-y\right|}{\rho(x)}\right)^{N}} \frac{|f(y)|}{\left|x_{0}-y\right|^{n-2}} \int_{B\left(y,\left|x_{0}-y\right| / 4\right)} \frac{V^{2}(z)}{|z-y|^{n-2}} d z d y \\
= & L_{1}+L_{2} .
\end{aligned}
$$

Note that

$$
\begin{aligned}
\int_{2^{k+1} B}\left|b(y)-b_{2 B} \| f(y)\right| d y & \lesssim \int_{2^{k+1} B}\left|b(y)-b_{2^{k+1} B} \| f(y)\right| d y+\left|b_{2^{k+1} B}-b_{2 B}\right| \int_{2^{k+1} B}|f(y)| d y \\
& \lesssim[b]_{\theta} k\left(1+\frac{2^{k} r}{\rho\left(x_{0}\right)}\right)^{\theta^{\prime}}\left(2^{k} r\right)^{\frac{n}{p^{\prime}}}\|f\|_{L^{p}\left(B\left(x_{0}, 2^{k+1} r\right)\right)}
\end{aligned}
$$


Then, by Lemma 2.3 we get

$$
\begin{aligned}
\mathrm{L}_{1} & \lesssim[\mathrm{b}]_{\theta} \sum_{k=1}^{\infty} \frac{\mathrm{k}}{\left(1+\frac{2^{\mathrm{k}} \mathrm{r}}{\rho\left(x_{0}\right)}\right)^{\mathrm{N} /\left(\mathrm{k}_{0}+1\right)-\theta^{\prime}}}\left(2^{\mathrm{k}} \mathrm{r}\right)^{-\frac{\mathfrak{n}}{\mathfrak{p}}}\|f\|_{L^{p}\left(B\left(x_{0}, 2^{k+1} r\right)\right)} \\
& \lesssim[b]_{\theta} \sum_{k=1}^{\infty} k\left(2^{k} r\right)^{-\frac{\mathfrak{n}}{\mathfrak{p}}}\|f\|_{L^{p}\left(B\left(x_{0}, 2^{k+1} r\right)\right)} \\
& \lesssim[b]_{\theta} \sum_{k=1}^{\infty} k \int_{2^{k} r}^{2^{k+1} r} \frac{\|f\|_{L^{p}\left(B\left(x_{0}, t\right)\right)}}{t^{\frac{n}{p}}} \frac{d t}{t}
\end{aligned}
$$

Note that $2^{k} r \leqslant t<2^{k+1} r$, then $k \approx \ln \frac{t}{r}$. Thus,

$$
\begin{aligned}
\mathrm{L}_{1} & \lesssim[\mathrm{b}]_{\theta} \sum_{k=1}^{\infty} \int_{2^{k} r}^{2^{k+1} r} \ln \frac{\mathrm{t}}{\mathrm{r}} \frac{\|f\|_{L^{p}\left(B\left(x_{0}, t\right)\right)}}{t^{\frac{n}{p}}} \frac{d t}{t} \\
& \lesssim[b]_{\theta} \int_{2 r}^{\infty}\left(1+\ln \frac{t}{r}\right) \frac{\|f\|_{L^{p}\left(B\left(x_{0}, t\right)\right)}}{t^{\frac{n}{p}}} \frac{d t}{t} .
\end{aligned}
$$

Choosing $\tilde{p}$ and $\tilde{s}$ such that $p>\tilde{p}$, and $1 / \tilde{p}^{\prime}=2 / \tilde{s}-2 / n$, then

$$
\begin{aligned}
& \mathrm{L}_{2} \lesssim \sum_{\mathrm{k}=1}^{\infty} \frac{1}{\left(2^{\mathrm{k}+1} \mathrm{r}\right)^{\mathrm{n}-2}} \frac{1}{\left(1+\frac{2^{\mathrm{k} r}}{\rho\left(x_{0}\right)}\right)^{\mathrm{N} /\left(\mathrm{k}_{0}+1\right)}} \\
& \times \int_{2^{k+1} B}\left|b(y)-b_{2 B} \| f(y)\right| I_{2}\left(V^{2} \chi_{B\left(x_{0}, 2^{k+1}\right)}\right)(y) d y \\
& \lesssim \sum_{k=1}^{\infty} \frac{1}{\left(2^{k+1} r\right)^{n-2}} \frac{1}{\left(1+\frac{2^{k} r}{\rho\left(x_{0}\right)}\right)^{N /\left(k_{0}+1\right)}} \\
& \times\left\|\left(b-b_{2 B}\right) f\right\|_{L^{\tilde{p}}\left(B\left(x_{0}, 2^{k+1} r\right)\right)}\left\|I_{2}\left(V^{2} \chi_{B\left(x_{0}, 2^{k+3} r\right)}\right)\right\|_{L^{\tilde{p}^{\prime}}\left(\mathbb{R}^{n}\right)} .
\end{aligned}
$$

Since $I_{2}$ from $L^{\tilde{s} / 2}$ to $L^{\tilde{p}^{\prime}}$ is bounded, and $V \in R H_{\tilde{s}}$, by (3.2) we have

$$
\left\|I_{2}\left(V^{2} \chi_{B\left(x_{0}, 2^{k+3} r\right)}\right)\right\|_{L_{\tilde{p}^{\prime}}\left(\mathbb{R}^{n}\right)} \lesssim\left(2^{k+1} r\right)^{\frac{n}{\tilde{p}^{\prime}}-2}\left(1+\frac{2^{k+1} r}{\rho\left(x_{0}\right)}\right)^{2 l_{0}} .
$$

Let $v=\frac{p \tilde{p}}{p-\tilde{p}}$, then

$$
\left\|\left(b-b_{2 B}\right) f\right\|_{L^{\tilde{p}}\left(B\left(x_{0}, 2^{k+1} r\right)\right)} \lesssim\|f\|_{L^{p}\left(B\left(x_{0}, 2^{k+1} r\right)\right)}\left\|b-b_{2 B}\right\|_{L^{v}\left(B\left(x_{0}, 2^{k+1} r\right)\right)}
$$

But

$$
\left\|\left(b-b_{2 B}\right)\right\|_{L^{v}\left(B\left(x_{0}, 2^{k+1} r\right)\right)} \lesssim[b]_{\theta} k\left|2^{k+1} B\right|^{\frac{1}{\tilde{p}}-\frac{1}{p}}\left(1+\frac{2^{k} r}{\rho\left(x_{0}\right)}\right)^{\theta^{\prime}} .
$$

Then

$$
\begin{aligned}
\mathrm{L}_{2} & \lesssim \sum_{\mathrm{k}=1}^{\infty} \frac{[\mathrm{b}]_{\theta} \mathrm{k}}{\left(1+\frac{2^{\mathrm{k} r}}{\rho\left(x_{0}\right)}\right)^{\mathrm{N} /\left(\mathrm{k}_{0}+1\right)-2 \mathrm{l}_{0}-\theta^{\prime}}}\left(2^{\mathrm{k}+1} \mathrm{r}\right)^{-\frac{\mathfrak{n}}{\mathfrak{p}}}\|f\|_{L^{p}\left(B\left(x_{0}, 2^{k+1} r\right)\right)} \\
& \lesssim[b]_{\theta} \int_{2 r}^{\infty}\left(1+\ln \frac{\mathrm{t}}{\mathrm{r}}\right) \frac{\|f\|_{L^{p}\left(B\left(x_{0}, t\right)\right)}}{t^{\frac{n}{p}}} \frac{d t}{t}
\end{aligned}
$$


Thus,

$$
\left\|\mathcal{R}^{*}\left(\left(b-b_{2 B}\right) f_{2}\right)\right\|_{L^{p}\left(B\left(x_{0}, r\right)\right)} \lesssim[b]_{\theta} r^{\frac{n}{p}} \int_{2 r}^{\infty}\left(1+\ln \frac{t}{r}\right) \frac{\|f\|_{L^{p}\left(B\left(x_{0}, t\right)\right)}}{t^{\frac{n}{p}}} \frac{d t}{t} .
$$

Combining (4.1), (4.2) and (4.3), the proof of Theorem 4.1 is completed.

Proof of Theorem 1.6. Since $f \in M_{p_{1}, \varphi_{1}}^{\alpha, V}$, and $\left(\varphi_{1}, \varphi_{2}\right)$ satisfies the condition (1.2), by (3.4) we have

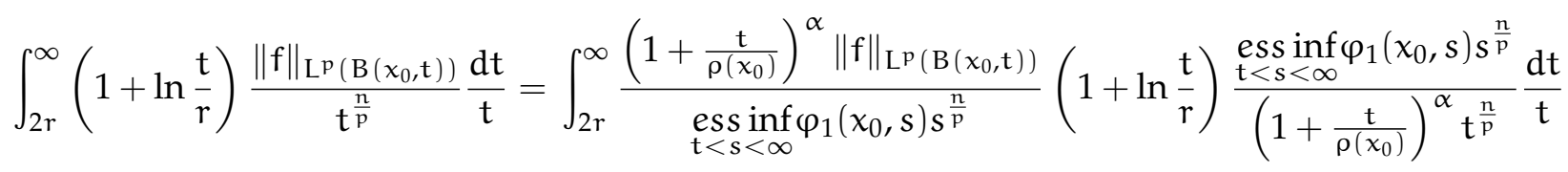

$$
\begin{aligned}
& \lesssim\|f\|_{M_{\mathcal{p}, \varphi_{1}}^{\alpha, v}} \int_{2 r}^{\infty}\left(1+\ln \frac{\mathrm{t}}{\mathrm{r}}\right) \frac{\begin{array}{l}
\operatorname{essinf} \varphi_{1}\left(x_{0}, s\right) s^{\frac{\mathfrak{n}}{p}} \\
\mathrm{t}<s<\infty
\end{array}}{\left(1+\frac{\mathrm{t}}{\rho\left(x_{0}\right)}\right)^{\alpha} t^{\frac{n}{p}}} \frac{d t}{t}
\end{aligned}
$$

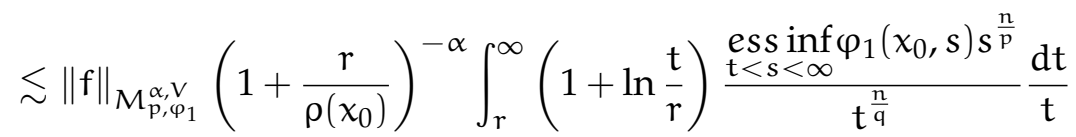

$$
\begin{aligned}
& \lesssim\|f\|_{M_{\mathcal{P}, \varphi_{1}}^{\alpha, V}}\left(1+\frac{r}{\rho\left(x_{0}\right)}\right)^{-\alpha} \varphi_{2}\left(x_{0}, r\right) .
\end{aligned}
$$

Then from Theorem 4.1 we get

$$
\begin{aligned}
\left\|\left[b, \mathcal{R}^{*}\right](f)\right\|_{M_{p, \varphi_{2}}^{\alpha, V}} & \lesssim \sup _{x_{0} \in \mathbb{R}^{n}, r>0}\left(1+\frac{r}{\rho\left(x_{0}\right)}\right)^{\alpha} \varphi_{2}\left(x_{0}, r\right)^{-1} r^{-n / p}\left\|\left[b, \mathcal{R}^{*}\right](f)\right\|_{L^{p}\left(B\left(x_{0}, r\right)\right)} \\
& \lesssim[b]_{\theta} \sup _{x_{0} \in \mathbb{R}^{n}, r>0}\left(1+\frac{r}{\rho\left(x_{0}\right)}\right)^{\alpha} \varphi_{2}\left(x_{0}, r\right)^{-1} \int_{2 r}^{\infty}\left(1+\ln \frac{t}{r}\right) \frac{\|f\|_{L^{p}\left(B\left(x_{0}, t\right)\right)}}{t^{\frac{n}{p}}} \frac{d t}{t} \\
& \lesssim[b]_{\theta}\|f\|_{M_{\mathcal{P}^{\prime}, \varphi_{1}}^{\alpha, V}}
\end{aligned}
$$

\section{References}

[1] B. Bongioanni, E. Harboure, O. Salinas, Commutators of Riesz transforms related to Schrödinger operators, J. Fourier Anal. Appl., 17 (2011), 115-134. 1, 2.2

[2] D. X. Chen, F. T. Jin, The boundedness of Marcinkiewicz integrals associated with Schrödinger operator on Morrey spaces, J. Funct. Spaces, 2014 (2014), 11 pages. 1, 1.4

[3] J. Dziubański, J. Zienkiewicz, $\mathrm{H}^{\mathrm{p}}$ spaces associated with Schrödinger operators with potentials from reverse Hölder classes, Colloq. Math., 98 (2003), 5-38.2.1

[4] Y. Liu, J. F. Dong, Some estimates of higher order Riesz transform related to Schrödinger type operators, Potential Anal., 32 (2010), 41-55. 1, 2.4

[5] Y. Liu, L. J. Wang, Boundedness for Riesz transform associated with Schrödinger operators and its commutator on weighted Morrey spaces related to certain nonnegative potentials, J. Inequal. Appl., 2014 (2014), 16 pages. 1

[6] Y. Liu, J. Zhang, J.-L. Sheng, L.-J. Wang, Some estimates for commutators of Riesz transform associated with Schrödinger operators, Czechoslovak Math. J., 66 (2016), 169-191. 1, 2.5, 2

[7] T. Mizuhara, Boundedness of some classical operators on generalized Morrey spaces, Harmonic analysis, ICM-90 Satell. Conf. Proc., Springer, Japan, (1991), 183-189. 1.4

[8] C. B. Jr. Morrey, On the solutions of quasi-linear elliptic partial differential equations, Trans. Amer. Math. Soc., 43 (1938), 126-166. 1, 1.4

[9] G. Pan, L. Tang, Boundedness for some Schrödinger type operators on weighted Morrey spaces, J. Funct. Spaces, 2014 (2014), 10 pages. 1

[10] Z. Shen, $\mathrm{L}^{\mathfrak{p}}$ estimates for Schrödinger operators with certain potentials, Ann. Inst. Fourier (Grenoble), 45 (1995), $513-$ 546. 1

[11] L. Tang, J. F. Dong, Boundedness for some Schrödinger type operator on Morrey spaces related to certain nonnegative potentials, J. Math. Anal. Appl., 355 (2009), 101-109. 1, 1.4

[12] R. L. Wheeden, A. Zygmund, Measure and integral: An introduction to real analysis, Marcel Dekker Inc., New York, (1977). 2.6 